\title{
Variation of parameters for local fractional nonhomogenous linear-differential equations
}

\author{
Mohammed AL Horani ${ }^{\mathrm{a}, \mathrm{b}}$, Mamon Abu Hammad ${ }^{\mathrm{b}}$, Roshdi Khalil ${ }^{\mathrm{b}, *}$ \\ ${ }^{a}$ Department of Mathematics, Faculty of Science, University of Hail, Saudi Arabia. \\ ${ }^{b}$ Department of Mathematics, The University of Jordan, Amman, Jordan.
}

\begin{abstract}
In this paper we study the method of variation of parameters to find a particular solution of a nonhomogenous linear fractional differential equations. A formula similar to that for usual ordinary differential equations is obtained. (C)2016 All rights reserved.

Keywords: Conformable fractional derivative, fractional integral, fractional differential equation, variation of parameters.

$2010 M S C: 26 \mathrm{~A} 33$.
\end{abstract}

\section{Introduction}

There are many definitions available in the literature for fractional derivatives. The main ones are the Riemann Liouville definition and the Caputo definition, see [10, 11], and for some applications one can see [5], [8] and [12].

(i) Riemann - Liouville Definition. For $\alpha \in[n-1, n)$, the $\alpha$ derivative of $f$ is

$$
D_{a}^{\alpha}(f)(t)=\frac{1}{\Gamma(n-\alpha)} \frac{d^{n}}{d t^{n}} \int_{a}^{t} \frac{f(x)}{(t-x)^{\alpha-n+1}} d x .
$$

${ }^{*}$ Corresponding author

Email addresses: m.alhorani@uoh.edu.sa (Mohammed AL Horani), roshdi@ju.edu.jo (Roshdi Khalil) 
(ii) Caputo Definition. For $\alpha \in[n-1, n)$, the $\alpha$ derivative of $f$ is

$$
D_{a}^{\alpha}(f)(t)=\frac{1}{\Gamma(n-\alpha)} \int_{a}^{t} \frac{f^{(n)}(x)}{(t-x)^{\alpha-n+1}} d x .
$$

Such definitions have many setbacks such as:

(i) The Riemann-Liouville derivative does not satisfy $D_{a}^{\alpha}(1)=0\left(D_{a}^{\alpha}(1)=0\right.$ for the Caputo derivative), if $\alpha$ is not a natural number.

(ii) All fractional derivatives do not satisfy the known formula of the derivative of the product of two functions:

$$
D_{a}^{\alpha}(f g)=f D_{a}^{\alpha}(g)+g D_{a}^{\alpha}(f) .
$$

(iii) All fractional derivatives do not satisfy the known formula of the derivative of the quotient of two functions:

$$
D_{a}^{\alpha}(f / g)=\frac{g D_{a}^{\alpha}(f)-f D_{a}^{\alpha}(g)}{g^{2}} .
$$

(iv) All fractional derivatives do not satisfy the chain rule:

$$
D_{a}^{\alpha}(f \circ g)(t)=f^{(\alpha)}(g(t)) g^{(\alpha)}(t) .
$$

(v) All fractional derivatives do not satisfy: $D^{\alpha} D^{\beta} f=D^{\alpha+\beta} f$, in general.

(vi) All fractional derivatives, specially Caputo definition, assumes that the function $f$ is differentiable.

In [9], the authors gave a new definition of fractional derivative which is a natural extension to the usual first derivative as follows:

Given a function $f:[0, \infty) \longrightarrow \mathbb{R}$. Then for all $t>0, \alpha \in(0,1)$, let

$$
T_{\alpha}(f)(t)=\lim _{\varepsilon \rightarrow 0} \frac{f\left(t+\varepsilon t^{1-\alpha}\right)-f(t)}{\varepsilon}
$$

$T_{\alpha}$ is called the conformable fractional derivative of $f$ of order $\alpha$.

Let $f^{(\alpha)}(t)$ stands for $T_{\alpha}(f)(t)$.

If $f$ is $\alpha$-differentiable in some $(0, b), b>0$ and $\lim _{t \rightarrow 0^{+}} f^{(\alpha)}(t)$ exists, then define

$$
f^{(\alpha)}(0)=\lim _{t \rightarrow 0^{+}} f^{(\alpha)}(t)
$$

According to this definition, we have the following properties, see [9],

1. $T_{\alpha}(1)=0$,

2. $T_{\alpha}\left(t^{p}\right)=p t^{p-\alpha}$ for all $p \in \mathbb{R}$,

3. $T_{\alpha}(\sin a t)=a t^{1-\alpha} \cos a t, a \in \mathbb{R}$,

$4 . T_{\alpha}(\cos a t)=-a t^{1-\alpha} \sin a t, a \in \mathbb{R}$, 


$$
5 . T_{\alpha}\left(e^{a t}\right)=a t^{1-\alpha} e^{a t}, a \in \mathbb{R} .
$$

Further, many functions behave as in the usual derivative. Here are some formulas

$$
\begin{aligned}
& T_{\alpha}\left(\frac{1}{\alpha} t^{\alpha}\right)=1, \\
& T_{\alpha}\left(e^{\frac{1}{\alpha} t^{\alpha}}\right)=e^{\frac{1}{\alpha} t^{\alpha}}, \\
& T_{\alpha}\left(\sin \frac{1}{\alpha} t^{\alpha}\right)=\cos \left(\frac{1}{\alpha} t^{\alpha}\right), \\
& T_{\alpha}\left(\cos \frac{1}{\alpha} t^{\alpha}\right)=-\sin \left(\frac{1}{\alpha} t^{\alpha}\right) .
\end{aligned}
$$

For more applications on the conformable fractional derivative, one can see [1, 2, 3, 4, 6, 7].

In this paper we use the conformable fractional derivative to study methods for finding particular solutions of a certain class of nonhomogenous linear fractional differential equations.

\section{Fractional linear differential equations}

Definition 2.1. Let $0<\alpha<1$ and $n \in\{1,2,3, \ldots\}$. Then we write

$$
T^{n \alpha} f=\underbrace{D^{\alpha} D^{\alpha} \ldots D^{\alpha}}_{\text {ntimes }} f \text {. }
$$

For simplicity, we write $f^{(n \alpha)}$ for $T^{n \alpha}$. So $y^{(2 \alpha)}(x)$ stands for $\frac{d^{\alpha}}{d x^{\alpha}}\left(\frac{d^{\alpha} y}{d x^{\alpha}}\right)$.

Definition 2.2. A differential equation of the form

$$
T^{n \alpha} y+a_{n-1} T^{(n-1) \alpha} y+\ldots+a_{1} T^{\alpha} y+a_{0} y=f(x)
$$

is called a linear fractional differential equation of order $n$. The coefficients $a_{0}, a_{1}, \ldots, a_{n-1}$ could be constants or variables.

Since $0<\alpha<1$, if $y$ is $n$-times differentiable, then there are $n$-independent solutions $y_{1}, y_{2}, \ldots, y_{n}$ for the homogeneous differential equation

$$
T^{n \alpha}+\ldots+a_{0} y=0 .
$$

To find a particular solution for (2.1), one can use either undetermined coefficients (for special types of $f$ and constant coefficients) or the variation of parameters method. In fact we find a complete formula for $y_{p}$ when $n=2$.

Definition 2.3. Let $y_{1}, y_{2}$ be two independent functions. The function

$$
W^{\alpha}\left[y_{1}, y_{2}\right]=\left|\begin{array}{cc}
y_{1} & y_{2} \\
y_{1}^{(\alpha)} & y_{2}^{(\alpha)}
\end{array}\right|
$$

will be called the $\alpha$-Wronskian of $y_{1}$ and $y_{2}$.

More generally, If $y_{1}, y_{2}, \ldots, y_{n}$ are $n$ linearly independent functions, then

$$
W^{\alpha}\left[y_{1}, y_{2}, \ldots, y_{n}\right]=\left|\begin{array}{cccc}
y_{1} & y_{2} & \ldots & y_{n} \\
y_{1}^{(\alpha)} & y_{2}^{(\alpha)} & \ldots & y_{n}^{(\alpha)} \\
\cdot & & & \\
\cdot & & & \\
\cdot & & & \\
y_{1}^{((n-1) \alpha)} & y_{2}^{((n-1) \alpha)} & \ldots & y_{n}^{((n-1) \alpha)}
\end{array}\right| \text {. }
$$




\section{The Main Results}

Let $y_{1}$ and $y_{2}$ be two independent solutions for

$$
D^{\alpha}\left(D^{\alpha} y\right)+a_{1} D^{\alpha} y+a_{0} y=0 .
$$

Our goal is to find $y_{p}$ for

$$
D^{\alpha}\left(D^{\alpha} y\right)+a_{1} D^{\alpha} y+a_{0} y=f(x) .
$$

Procedure:

Step(i). Let

$$
y_{p}=c_{1} y_{1}+c_{2} y_{2}
$$

where $c_{1}$ and $c_{2}$ are functions of $x$. So

$$
\begin{gathered}
y_{p}(x)=c_{1}(x) y_{1}(x)+c_{2}(x) y_{2}(x), \\
y_{p}^{(\alpha)}=c_{1}^{(\alpha)} y_{1}+c_{1} y_{1}^{(\alpha)}+c_{2}^{(\alpha)} y_{2}+c_{2} y_{2}^{(\alpha)} .
\end{gathered}
$$

Step(ii). Put

$$
c_{1}^{(\alpha)} y_{1}+c_{2}^{(\alpha)} y_{2}=0
$$

Hence

$$
\begin{aligned}
D^{\alpha}\left(y_{p}^{(\alpha)}\right) & =D^{\alpha}\left(c_{1} y_{1}+c_{2} y_{2}\right) \\
& =c_{1}^{(\alpha)} y_{1}^{(\alpha)}+c_{1} y_{1}^{(2 \alpha)}+c_{2}^{(\alpha)} y_{2}^{(\alpha)}+c_{2} y_{2}^{(2 \alpha)} .
\end{aligned}
$$

Step(iii). Substitute $D^{\alpha}\left(y_{p}^{(\alpha)}\right)$ in the equation 3.2 to get

$$
c_{1}^{(\alpha)} y_{1}^{(\alpha)}+c_{1} y_{1}^{(2 \alpha)}+c_{2}^{(\alpha)} y_{2}^{(\alpha)}+c_{2} y_{2}^{(2 \alpha)}+a_{1}\left(c_{1} y_{1}^{(\alpha)}+c_{2} y_{2}^{(\alpha)}\right)+a_{0}\left(c_{1} y_{1}+c_{2} y_{2}\right)=f(x) .
$$

Hence,

$$
c_{1}\left(y_{1}^{(2 \alpha)}+a_{1} y_{1}^{(\alpha)}+a_{0} y_{1}\right)+c_{2}\left(y_{2}^{(2 \alpha)}+a_{1} y_{2}^{(\alpha)}+a_{0} y_{2}\right)+c_{1}^{(\alpha)} y_{1}^{(\alpha)}+c_{2}^{(\alpha)} y_{2}^{(\alpha)}=f(x) .
$$

Step(iv). Since $y_{1}$ and $y_{2}$ are solutions for 3.2 , we get

$$
c_{1}^{(\alpha)} y_{1}^{(\alpha)}+c_{2}^{(\alpha)} y_{2}^{(\alpha)}=f(x) .
$$

Now we have to solve 3.3$)$ and $(3.4)$ to get

$$
c_{1}^{(\alpha)}=-\frac{f(x) y_{2}(x)}{W^{\alpha}\left[y_{1}, y_{2}\right]}
$$

and

Thus

$$
c_{2}^{(\alpha)}=\frac{f(x) y_{1}(x)}{W^{\alpha}\left[y_{1}, y_{2}\right]} .
$$

$$
y_{p}(x)=\int_{a}^{x} \frac{\left|\begin{array}{cc}
y_{1}(t) & y_{2}(t) \\
y_{1}(x) & y_{2}(x)
\end{array}\right|}{W^{\alpha}\left[y_{1}, y_{2}\right](t)} \frac{f(t)}{t^{1-\alpha}} d t
$$


that is to say,

$$
\begin{aligned}
y_{p}(x) & =I_{\alpha}^{a}\left[\frac{\left|\begin{array}{cc}
y_{1}(t) & y_{2}(t) \\
y_{1}(x) & y_{2}(x)
\end{array}\right|}{W^{\alpha}\left[y_{1}, y_{2}\right](t)} f(t)\right] \\
& =I_{\alpha}^{a}(K(x, t) f(t)),
\end{aligned}
$$

where

$$
K(x, t)=\frac{\left|\begin{array}{cc}
y_{1}(t) & y_{2}(t) \\
y_{1}(x) & y_{2}(x)
\end{array}\right|}{W^{\alpha}\left[y_{1}, y_{2}\right](t)}
$$

Similarly we can consider the case of higher order linear fractional differential equations. Let $y_{1}, y_{2}, \ldots, y_{n}$ be linearly independent solutions of the homogeneous equation (2.2). As we have shown above, one can find a particular solution of the nonhomogeneous equation (2.1) of the form

$$
y_{p}(x)=c_{1}(x) y_{1}(x)+c_{2}(x) y_{2}(x)+\ldots+c_{n}(x) y_{n}(x) .
$$

One can apply the same steps in the previous process and obtain the following nonhomogeneous algebraic linear system of $n$ equations for $c_{1}^{(\alpha)}, c_{2}^{(\alpha)}, \ldots, c_{n}^{(\alpha)}$

$$
\begin{aligned}
& y_{1} c_{1}^{(\alpha)}+y_{2} c_{2}^{(\alpha)}+\ldots+y_{n} c_{n}^{(\alpha)}=0 \\
& y_{1}^{(\alpha)} c_{1}^{(\alpha)}+y_{2}^{(\alpha)} c_{2}^{(\alpha)}+\ldots+y_{n}^{(\alpha)} c_{n}^{(\alpha)}=0 \\
& y_{1}^{(2 \alpha)} c_{1}^{(\alpha)}+y_{2}^{(2 \alpha)} c_{2}^{(\alpha)}+\ldots+y_{n}^{(2 \alpha)} c_{n}^{(\alpha)}=0 \\
& \vdots \\
& y_{1}^{((n-1) \alpha)} c_{1}^{(\alpha)}+y_{2}^{((n-1) \alpha)} c_{2}^{(\alpha)}+\ldots+y_{n}^{((n-1) \alpha)} c_{n}^{(\alpha)}=f .
\end{aligned}
$$

Using Cramer's rule we find

$$
c_{m}^{(\alpha)}(x)=\frac{f(x) W_{m}^{\alpha}(x)}{W^{\alpha}(x)}
$$

where $W^{\alpha}(x)=W^{\alpha}\left(y_{1}, y_{2}, \ldots, y_{n}\right)(x)$ and $W_{m}^{\alpha}$ is the determinant obtained from $W^{\alpha}$ by replacing the mth column by the column $(0,0, \ldots, 0,1)$. Thus

$$
y_{p}(x)=\sum_{m=1}^{n} y_{m} \int_{a}^{x} \frac{f(t) W_{m}^{\alpha}(t)}{W^{\alpha}(t) t^{1-\alpha}} d t
$$

where $a$ is an arbitrary positive constant.

\section{Examples}

Example 4.1. We first solve the following fractional differential equation

$$
D^{1 / 2}\left(D^{1 / 2} y\right)=\ln x, \quad x>0 .
$$


One can easily show that $y_{1}=1$ and $y_{2}=\sqrt{x}$ are two linearly independent solutions of the corresponding homogeneous equation

$$
D^{1 / 2}\left(D^{1 / 2} y\right)=0 .
$$

Let us find the particular solution $y_{p}(x)$ of equation (4.1) using the method of variation of parameters, precisely, we want to apply the formula

$$
\begin{aligned}
y_{p}(x) & =-y_{1} \int \frac{y_{2} f(x)}{W^{\alpha}\left[y_{1}, y_{2}\right] x^{1-\alpha}} d x+y_{2} \int \frac{y_{1} f(x)}{W^{\alpha}\left[y_{1}, y_{2}\right] x^{1-\alpha}} d x \\
& =-y_{1} \int \frac{y_{2} f(x)}{W\left[y_{1}, y_{2}\right] x^{2-2 \alpha}} d x+y_{2} \int \frac{y_{1} f(x)}{W\left[y_{1}, y_{2}\right] x^{2-2 \alpha}} d x .
\end{aligned}
$$

Since $y_{1}=1, y_{2}=\sqrt{x}, f(x)=\ln x$ and $W\left[y_{1}, y_{2}\right]=\frac{1}{2 \sqrt{x}}$, we obtain

$$
\begin{aligned}
y_{p}(x) & =-\int \frac{\sqrt{x} \ln x}{\frac{1}{2 \sqrt{x}} x} d x+\sqrt{x} \int \frac{\ln x}{\frac{1}{2 \sqrt{x}} x} d x \\
& =-2 \int \ln x d x+2 \sqrt{x} \int \frac{\ln x}{\sqrt{x}} d x \\
& =2 x \ln x-6 x .
\end{aligned}
$$

Thus, the general solution $y(x)$ can be written as

$$
y(x)=c_{1}+c_{2} \sqrt{x}+2 x \ln x-6 x .
$$

Example 4.2. Consider the following fractional differential equation

$$
x D^{1 / 2}\left(D^{1 / 2} y\right)-\frac{1}{2} y=x^{3 / 2}+x, \quad x>0 .
$$

One can easily show that $y_{1}=x$ and $y_{2}=x^{-1 / 2}$ are two linearly independent solutions of the corresponding homogeneous equation

$$
x D^{1 / 2}\left(D^{1 / 2} y\right)-\frac{1}{2} y=0 .
$$

Let us find the particular solution $y_{p}(x)$ of equation (4.3) using the formula

$$
y_{p}(x)=-y_{1} \int \frac{y_{2} f(x)}{W\left[y_{1}, y_{2}\right] x^{2-2 \alpha}} d x+y_{2} \int \frac{y_{1} f(x)}{W\left[y_{1}, y_{2}\right] x^{2-2 \alpha}} d x \text {. }
$$

Substitute $y_{1}=x, y_{2}=x^{-1 / 2}, f(x)=x^{1 / 2}+1$ and $W\left[y_{1}, y_{2}\right]=-\frac{3}{2} x^{-1 / 2}$ in the above equation, we get

$$
\begin{aligned}
y_{p}(x) & =-x \int \frac{x^{-1 / 2}\left(x^{1 / 2}+1\right)}{-\frac{3}{2} x^{-1 / 2} x} d x+x^{-1 / 2} \int \frac{x\left(x^{1 / 2}+1\right)}{-\frac{3}{2} x^{-1 / 2} x} d x \\
& =x^{3 / 2}+\frac{2}{3} x \ln x-\frac{4}{9} x .
\end{aligned}
$$

Thus the general solution of equation 4.3 can be written as

$$
y(x)=c_{1} x+c_{2} x^{-1 / 2}+x^{3 / 2}+\frac{2}{3} x \ln x-\frac{4}{9} x .
$$


Example 4.3. Let us solve the following fractional differential equation

$$
D^{1 / 2}\left(D^{1 / 2}\left(D^{1 / 2} y\right)\right)=x^{5 / 2} .
$$

One can verify that $y_{1}=1, y_{2}=x$ and $y_{3}=x^{1 / 2}$ are linearly independent solutions of the homogeneous equation

$$
D^{1 / 2}\left(D^{1 / 2}\left(D^{1 / 2} y\right)\right)=0 .
$$

By simple calculations, we can obtain

$$
W^{1 / 2}=-\frac{1}{4}, \quad W_{1}^{1 / 2}=-\frac{1}{2} x, \quad W_{2}^{1 / 2}=-\frac{1}{2} \quad \text { and } \quad W_{3}^{1 / 2}=x^{1 / 2} .
$$

Now, see (3.7),

$$
\begin{aligned}
y_{p}(x) & =y_{1} \int \frac{W_{1}^{1 / 2} f(x)}{W^{1 / 2} x^{1 / 2}} d x+y_{2} \int \frac{W_{2}^{1 / 2} f(x)}{W^{1 / 2} x^{1 / 2}} d x+y_{3} \int \frac{W_{3}^{1 / 2} f(x)}{W^{1 / 2} x^{1 / 2}} d x \\
& =\int \frac{-\frac{1}{2} x x^{5 / 2}}{-\frac{1}{4} x^{1 / 2}} d x+x \int \frac{-\frac{1}{2} x^{5 / 2}}{-\frac{1}{4} x^{1 / 2}} d x+x^{1 / 2} \int \frac{x^{1 / 2} x^{5 / 2}}{-\frac{1}{4} x^{1 / 2}} d x \\
& =2 \int x^{3} d x+2 x \int x^{2} d x-4 x^{1 / 2} \int x^{5 / 2} d x \\
& =\frac{1}{42} x^{4} .
\end{aligned}
$$

Thus the general solution of equation 4.5 is given by

$$
y(x)=c_{1}+c_{2} x+c_{3} x^{1 / 2}+\frac{1}{42} x^{4} .
$$

\section{References}

[1] T. Abdeljawad, On conformable fractional calculus, J. Comput. Appl. Math., 279 ( 2015), 57-66. 1

[2] T. Abdeljawad, M. Al Horani, R. Khalil, Conformable fractional semigroups of operators, J. Semigroup Theory Appl., 2015 (2015), 9 pages. 1

[3] B. Bayour, D. F. M. Torres, Existence of solution to a local fractional nonlinear differential equation, J. Comput. Appl. Math., (2016), (in press). 1

[4] N. Benkhettou, S. Hassani, D. F. M. Torres, A conformable fractional calculus on arbitrary time scales, J. King Saud Univ. Sci., 28 (2016), 93-98. 1

[5] T. Caraballoa, M. A. Diopb, A. A. Ndiayeb, Asymptotic behavior of neutral stochastic partial functional integro-differential equations driven by a fractional Brownian motion, J. Nonlinear Sci. Appl., 7 (2014), 407-421. 目

[6] A. Gökdoğan, E. Ünal, E. Çelik, Existence and uniqueness theorems for sequential linear conformable fractional differential equations, to appear in Miskolc Mathematical Notes.1]

[7] M. A. Hammad, R. Khalil, Abel's formula and Wronskian for conformable fractional differential equations, Int. J. Differ. Equ. Appl., 13 (2014), 177-183. 1

[8] M. Hao, C. Zhai, Application of Schauder fixed point theorem to a coupled system of differential equations of fractional order, J. Nonlinear Sci. Appl., 7 (2014), 131-137. 1

[9] R. Khalil, M. Al Horani, A. Yousef, M. Sababheh, A new definition of fractional derivative, J. Comput. Appl. Math., 264 (2014), 65-70. 1

[10] A. A. Kilbas, H. M. Srivastava, J. J. Trujillo, Theory and applications of fractional differential equations, Elsevier Science B.V., Amsterdam, (2006). 1

[11] K. S. Miller, B. Ross, An introduction to fractional calculus and fractional differential equations, John Wiley and Sons, New York, (1993). 1

[12] J. A. Nanware, D. B. Dhaigude, Existence and uniqueness of solutions of differential equations of fractional order with integral boundary conditions, J. Nonlinear Sci. Appl., 7 (2014), 246-254. 1 